\title{
The choice of sports affects mental rotation performance in adolescents
}

Stefanie Pietsch \& Petra Jansen, Institute of Sport Science, University of Regensburg.

\section{Introduction}

Physical activity causes positive effects on different parts of cognition. Especially, athletes exhibited superior performance in spatial tasks compared to physically inactive people (Pietsch \& Jansen, 2012; Voyer \& Jansen, 2017). However the influence of individual sports disciplines on different forms of mental rotation performance requires more precise research. This study investigates the specific relationship between different sports (soccer/dancing) and specific mental rotation tasks (egocentric/object-based) with embodied stimuli to specify the relation of motor and visuo-spatial processes of female adolescents.

\section{Method}

\section{Participants and Material}

28 female dancers (mean age $=15.54, S D=$ 1.13) and 28 female soccer players (mean age $=15.29, S D=1.35$ ) participated in this study. Neither differed the groups in the amount of years, they practice their specific sports, nor in sporting hours per week, cognitive processing speed and gender role identity.

\section{Mental Rotation Test}

The chronometric mental rotation task (Software "Presentation") contained an object-based and an egocentric stimulus condition with male and female stimul presented in front and back view with either the left or the right arm raised in eight different angular disparities. There were two blocks of object-based and egocentric condition, the order of the blocks was counterbalanced.

\section{Procedure/Statistical Analysis}

\section{Procedure}

Participants conducted a demographic questionnaire, followed by the Number Connection Test (Oswald \& Roth, 1987), the SCS, the BSRI and the CMRT (see Figure 1 and 2). The experiment contained 12 blocks of 32 experimental trials each (192 objectbased, 192 egocentric).

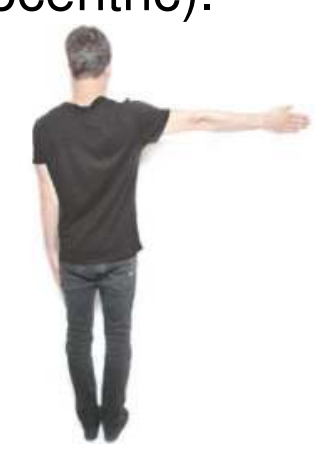

Figure 1: Egocentric condition (back)

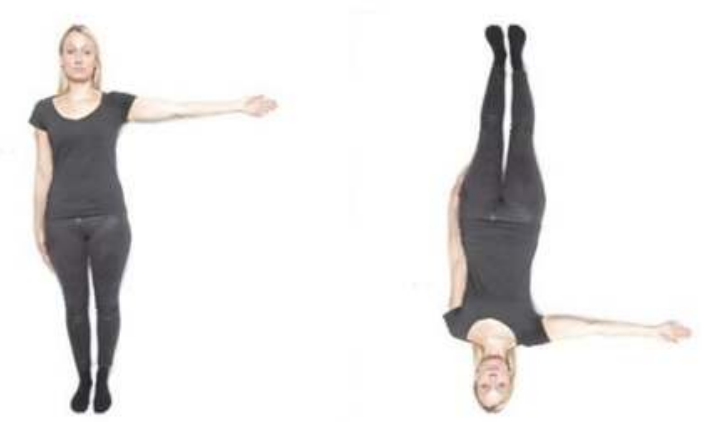

Figure 2: Object-based condition (front) Statistical Analysis

Two repeated measurement ANOVAs with "reaction time" and "accuracy rate" as dependent variables were conducted with the between subject factor "group" and the three within-subject factors "angular disparity" $\left(0^{\circ}\right.$, $45^{\circ}, 90^{\circ}, 135^{\circ}, 180^{\circ}, 225^{\circ}, 270^{\circ}, 315^{\circ}$, "view" (front vs. back), and "kind of transformation" (object-based vs. egocentric). The significant interactions were analysed further with t-tests. Due to the multipletesting, we alpha-corrected in line with Bonferroni, resulting in a corrected significance level of $p<.00625$ for the interaction with angular disparity.

\section{Results}

\section{Reaction time:}

Two-way interaction between

a) "kind of transformation" and "view":

$F(1,54)=4.306, p=.05$, partial $\eta 2=.074$

b) "view" and "group":

$F(1,54)=4.302, p<.05$, partial $\eta 2=.074$

Significant reaction time differences:

a) No differences in back view:

$t(54)=-.754, p=.454$

b) Front view: $\mathrm{t}(54)=-2.113, p<.05$

Soccer-players $(M=1251.49 \mathrm{~ms}, \quad S D=$ 347.78) showed shorter reaction times than dancers $(M=1527.99 \mathrm{~ms}, S D=425.16)$.

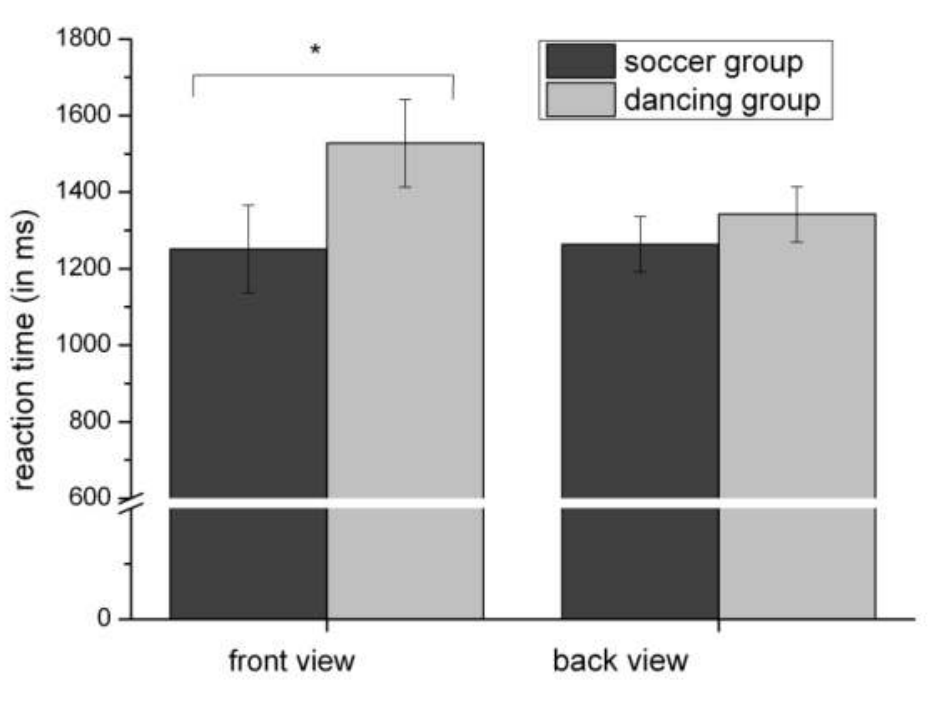

Figure 3: Reaction time

\section{Accuracy:}

Three-way interaction between

"kind of transformation", "view", and "group":

$F(1,54)=7.737, p<.005$, partial $\eta^{2}=.125$

Significant accuracy rate differences:

a) Object-based stimuli in back view $\mathrm{t}(54)=-3.654, \mathrm{p}=.001$

Dancers showed higher accuracy rates than soccer-players.

b) Object-based stimuli in front view: $t(54)=-1.870$, n.s.

There was no significant difference for egocentric transformations in front view, $\mathrm{t}(54)=-1.165$, n.s., and back view, $\mathrm{t}(54)=-$

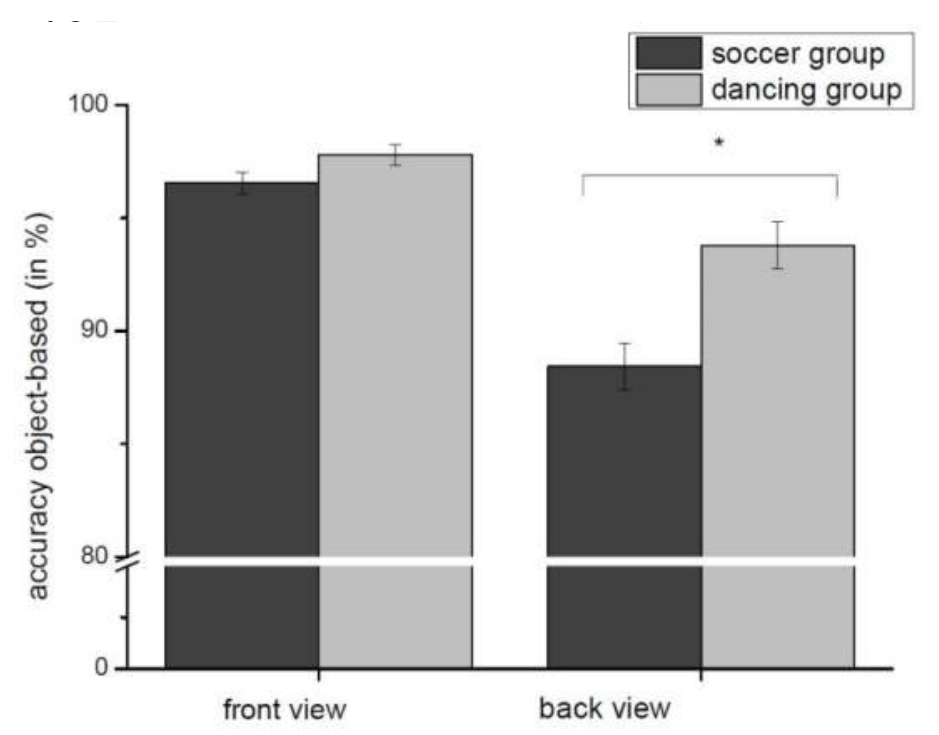

Figure 4: Accuracy object-based

\section{Discussion}

The results of this study indicate, that:

Specific aspects of mental rotation performance can be affected by different forms of physical activity.

Individual aspects of mental rotation performance depend on the sport- specific demands of visual perception.

Gender-stereotype sports have to be included in further research. 